\title{
EDITORIAL
}

\section{Detecting airways obstruction from the tidal flow profile}

\author{
J.H.T. Bates
}

The two papers in this issue from the Osler Chest Unit at Oxford University [1,2] exploit the fact that the breathing pattern in humans is affected in characteristic ways when the pulmonary airways are obstructed. MoRRIs et al. [1] examined the latter part of the tidal expiratory flow pattern in patients with varying degrees of airflow obstruction and found that the expiratory time constant correlated positively with standard measures of airflow obstruction. Williams et al. [2] also showed that a shape index related to the rate of decay of post-maximal expiratory flow correlated strongly with airflow obstruction, as determined by conventional methods. These studies suggest that a simple, noninvasive procedure may be used to derive an important physiological parameter that is not normally so easily obtained.

Assessing airflow obstruction directly means measuring airway or lung resistance, which requires subjecting the subject to a technique that is either cumbersome or invasive. For example, airway resistance (Raw) can be assessed by body plethysmography [3], but this requires a significant degree of subject cooperation and the ability to deal with claustrophobia. Lung resistance can be determined from flow at the mouth and the pressure drop across the lung [4], but this requires the placement of an oesophageal balloon, which is not a trivial matter and is out of the question for routine screening. Respiratory system impedance can be determined by the forced oscillation method [5], in which perturbations in flow are applied to the mouth by an oscillator. Although promising, this method is attended by numerous technical difficulties and has yet to be put to widespread use. Direct visualization of constricted airways is also possible, in principle, but at, the expense of the X-ray dose resulting from a computed tomographic scan [6].

The results of MorRIs et al. [1] and WilLIAMs et al. [2] are, therefore, interesting from a clinical perspective be-cause they suggest that the severity of airflow obstruction, a parameter of key concern to the respiratory specialist, may be derived easily and noninvasively, namely from airflow measurements at the mouth. The physiological basis of this approach is not difficult to appreciate. A spontaneously breathing individual indulges in a process of feedback control, whereby the respiratory centres in the brain direct the respiratory musculature to overcome the mechanical impedance of the lungs and thorax in such a fashion as to achieve the necessary alveolar ventilation. If the respiratory impedance increases, as occurs with airflow obstruction, the respiratory centres must obviously respond

Correspondence: J.H.T. Bates, Meakins-Christie Laboratories and Dept of Biomedical Engineering, McGill University, 3626 St Urbain Street, Montreal, Quebec, Canada H2X 2P2. Fax: 5143987483. by increasing drive to the muscles in order to maintain ventilation. However, the particular pattern of ventilation that results (i.e. the shape of the mouth flow profile over a breath) reflects the combined effects of increased amplitude and/or frequency of breathing efforts as well as the altered mechanical properties of the lungs. Humans and other animals increase their respiratory drive in response to various loads in characteristic ways [7]. Furthermore, airflow obstruction leads to characteristic changes in the way in which a given drive produces a particular kind of flow pattern (e.g. increased airway resistance increases the expiratory time constant of the respiratory system). Consequently, it is not surprising that airflow obstruction tends to lead to characteristic changes in the pattern of spontaneous breathing. MorRis et al. [1] and WiLLiams et al. [2] have tried to identify particular features of the expiratory airflow pattern that capture these characteristic changes.

However, care must be taken not to forget that airflow obstruction only tends to lead to characteristic changes in the pattern of spontaneous breathing. The latter is by no means a physiologically necessary sequel of the former. In other words, the observation of a statistically significant correlation between a feature of the breathing pattern and airflow obstruction does not mean that one can automatically take one as a surrogate for the other. There are at least three reasons for this. Firstly, a statistically significant correlation between two variables simply means that they have an apparent element of commonality between them. It does not mean that one can immediately be taken as an accurate estimate of the other. This would be the case only if the correlation between the two variables was precisely 1 . Therefore, the real issue one needs to consider is, if one is seeking variable $A$ how accurately does one estimate variable $A$ by measuring variable $B$, and is this accuracy sufficient for the purpose at hand? This question is not answered by the value of a correlation coefficient, but rather requires a consideration of the statistical reliability of determining $A$ from $B$, which can be addressed through standard regression theory [8].

The second reason why we must be cautious with indirect physiological measures is that they may be influenced by factors other than those in which we are interested. In the case of the breathing pattern, for example, the rate of decay of post-peak expiratory flow may be affected by the rate at which post-inspiratory muscle activity wanes [9] or by changes in glottic aperture [10], neither of which have any direct connection with the airflow obstruction in an asthmatic individual. It may be that, in most cases, these extraneous factors are not the dominant ones. Nevertheless, one has to remember that an indirect measure of airflow obstruction derived from the spontaneous breath- 
ing pattern may be significantly affected by something that has nothing to do with the quantity or process of interest.

The third reason why a parameter derived from the breathing pattern must be used with caution when investigating airflow obstruction is that it can be very difficult, if not impossible, to relate the parameter to the underlying processes causing the obstruction. In the case of $R$ aw, for example, the laws of physics draw an immediate connection between an elevated value for $R$ aw and a narrowed airway lumen [11]. In the case of an altered airflow pattern, however, the way in which simultaneous changes in both neural drive and lung mechanics interact to produce the resultant flow are generally not obvious, so an ob-served alteration in a breathing pattern may have many interpretations.

The analysis of the spontaneous breathing pattern as a means of assessing the mechanical properties of the lungs is also currently receiving considerable attention amongst animal experimenters. The widespread use of small rodents (rats and mice) in medical research extends to those investigating bronchopharmacology and various animal models of lung disease. The technical difficulties of measuring the mechanical properties of tiny rodent lungs, together with the widespread desire to make measurements in awake animals, has resulted in the popularization of a quantity known as the enhanced pause. This quantity is derived from the amplitude and timing of a signal produced by an animal breathing spontaneously in a leaky body plethysmograph. The enhanced pause has been shown to correlate empirically with airflow obstruction [12], but again the actual physiological connection between the enhanced pause and airflow obstruction is unclear and may have numerous causes. Consequently, while the enhanced pause and similar quantities can be useful in determining whether something interesting is going on in the lungs, the final resolution of issues involving airway narrowing or lung stiffening must be made by methods that measure the mechanical properties of the lungs directly and unambiguously.

Thus, the use of the spontaneous breathing pattern alone to derive an index of airflow obstruction must be viewed in its proper perspective. It is easy to apply, is completely noninvasive and requires no cooperation from the subject. As such, it has applicability to virtually all age ranges and circumstances. However, the spontaneous breathing pattern can never reveal the severity, or even the presence, of airflow obstruction with complete reliability, because there is always the chance that the spontaneous breathing pattern has been affected purely by an abnormality in the neural control of breathing. Nevertheless, the practical advantages of breathing pattern analysis are obvious and significant, suggesting that it may play a useful role as a first-line diagnostic tool in the investigation of airflow obstruction. Indeed, as more sophisticated and subtle signal processing methods are applied to the analysis of the spontaneous breathing pattern, it may reveal itself to be useful in the diagnosis of many pulmonary pathologies.

\section{References}

1. Morris MJ, Madgwick RG, Collyer I, Denby F, Lane DJ. Analysis of expiratory tidal flow patterns as a diagnostic tool in airflow obstruction. Eur Respir J 1998; 12: 11131117

2. Williams EM, Madgwick RG, Morris MJ. Expired airflow patterns in adults with airway obstruction. Eur Respir J 1998; 12: 1118-1123.

3. DuBois AB, Botelho SY, Conroe JH. A new method for measuring airway resistance in man using a body plethysmograph: values in normal subjects and in patients with respiratory disease. J Clin Invest 1956; 35: 327-335.

4. Mead J, Whittenberger JL. Physical properties of human lungs measured during spontaneous respiration. $J$ Appl Physiol 1953; 5: 779-796.

5. Peslin R, Fredberg JJ. Oscillation mechanics of the respiratory system. In: Macklem PT, Mead J, eds. Handbook of Physiology. Section 3. The Respiratory System, Vol. III. Mechanics of Breathing. Bethesda, MD, American Physiological Society, 1986; pp. 145-178.

6. Brown RH, Mitzner W, Wagner EM. Interaction between airway edema and lung inflation on responsiveness of individual airways in vivo. J Appl Physiol 1997; 83: 366370.

7. Milic-Emili J, Zin WA. Breathing responses to imposed mechanical loads. In: Cherniack NS, Widdicombe JG, eds. Handbook of Physiology. Section 3. The Respiratory System, Vol. II. Control of Breathing. Bethesda, MD, American Physiological Society, 1986, pp. 751-770.

8. Draper NR, Smith H. Applied Regression Analysis. New York, Wiley, 1966.

9. Shee CD, Ploy-Song-Sang Y, Milic-Emili J. Decay of inspiratory muscle pressure during expiration in conscious humans. J Appl Physiol 1985; 58: 1859-1865.

10. Séries F, Cormier Y, Desmeules M. Influence of passive changes of lung volume on upper airways. J Appl Physiol 1990; 68: 2159-2164.

11. Pedley TJ, Drazen JM. Aerodynamic theory. In: Macklem PT, Mead J, eds. Handbook of Physiology. Section 3. The Respiratory System. Vol. III. Mechanics of Breathing. Bethesda, MD, American Physiological Society, 1986; pp. 41-54.

12. Hamelmann E, Schwarze J, Takeda K, et al. Noninvasive measurement of airway responsiveness in allergic mice using barometric plethysmography. Am J Respir Crit Care Med 1997; 156: 766-775. 